\title{
Análisis pedagógico de los resultados de evaluación SNEPE 2015 en lengua castellana y literatura del tercer curso de la Media, y su correspondencia con la taxonomía de Bloom
}

\author{
Pablo Iván Paredes Mora \\ ivnparedes@hotmail.com \\ Doctorando en Ciencias de la Educación, Facultad de Humanidades y Ciencias de la Educación \\ (FHCE). Universidad Nacional de Pilar (UNP)
}

\section{RESUMEN}

Este trabajo se ubica en el ámbito de los resultados de la prueba SNEPE 2015 en lengua Castellana y Literatura del tercer curso de la Educación Media. Se ha realizado un análisis pedagógico de los resultados para saber cuáles fueron los motivos que llevaron a los estudiantes a no lograr las derivaciones esperadas. Las muestras fueron tomadas de la prueba SNEPE, donde se observa que los estudiantes del tercer curso de la Educación. Media de la educación paraguaya tuvieron resultados inferiores a lo que se pretendía. Fueron seleccionados contenidos de área evaluados, contrastando con los ejercicios que realizan los estudiantes en horas de clase para el desarrollo de la asignatura. Además de autores que discuten y actualizan los temas referentes a evaluación de los aprendizajes y taxonomía de Bloom, que propone dividir los objetivos cognitivos en subcategorías organizadas del comportamiento más simple al más complejo. Los resultados obtenidos han demostrados que los libros de texto de Lengua Castellana y Literatura de tercer curso de la Educación. Media que utilizan los estudiantes no condicen con los estilos de pruebas de las evaluaciones de SNEPE.

Palabras clave: Análisis pedagógico; Evaluación; Educación Media; Lengua Castellana; Taxonomía de Bloom. 


\title{
Pedagogical analysis of the SNEPE 2015 evaluation results in Spanish language and literature from the third year of the Media, and their correspondence with Bloom's taxonomy
}

\begin{abstract}
This work is located within the scope of the results of the SNEPE 2015 test in Spanish language and Literature of the third year of Secondary Education. A pedagogical analysis of the results has been carried out to find out what were the reasons that led the students not to achieve the expected referrals. The samples were taken from the SNEPE test, where it is observed that the students of the third year of Education. Average of Paraguayan education had lower results than what was intended. The contents of the evaluated area were selected, contrasting with the exercises that the students carry out in class hours for the development of the subject. In addition to authors who discuss and update the issues related to assessment of learning and Bloom's taxonomy, who proposes dividing cognitive objectives into organized subcategories from the simplest to the most complex behavior. The results obtained have shown that the textbooks of Spanish Language and Literature of the third year of Education. Average used by students does not match the testing styles of the SNEPE assessments.
\end{abstract}

Keywords: Pedagogical analysis; Evaluation; Middle education; Spanish language; Bloom's Taxonomy. 


\section{INTRODUCCIÓN}

Este trabajo analiza los resultados de la prueba SNEPE 2015 en lengua Castellana del tercer curso de la Media de la educación paraguaya. Son contrastados con autores que investigan y actualizan los cambios que tienen los conceptos de evaluación de los aprendizajes en la actualidad.

Tiene por objetivo demostrar que los libros de lengua Castellana y Literatura del Tercer curso de la Media utilizados por los estudiantes para desarrollar la asignatura tienen unos desaciertos. Los ejercicios para la fijación del conocimiento no son presentados conforme a las pruebas SNEPE, tampoco con los niveles de complejidades de la taxonomía de Bloom. Para realizar el análisis pedagógico de los resultados de la evaluación SNEPE 2015 y su relación con la taxonomía de Bloom. Tomamos como referencia el Informe Nacional por Eje Temático evaluación censal 2015. En ella. "La tabla de especificaciones establece los ejes temáticos y temas del programa curricular de los grados y áreas evaluados y determina el nivel de dificultad teórica de los ítems elaborados, que se basan en la Taxonomía de Bloom y abarcan diferentes procesos cognitivos" (p. 4).

Pero, qué es el SNEPE y cuanto surge. Según el Módulo I, uso de los resultados de las pruebas de aprendizaje del SNEPE en instituciones educativas (2016):

Se creó en abril de 1995 para el aprovechamiento de los resultados para la orientación del aprendizaje. En ese entonces, el Paraguay contaba con un sistema educativo sin cultura ni tradición en evaluación. Inicialmente, se optó por una cobertura muestral considerando los propósitos, la viabilidad financiera, y la posible resistencia por parte de los docentes a una evaluación censal. A partir de esa experiencia y con el reconocimiento de la importancia de las evaluaciones educativas como instrumento orientador para el mejoramiento de los logros académicos, en 2015 el SNEPE llevó a cabo una evaluación de tipo censal por primera vez en el país (p. 5).

Los resultados de las pruebas obtenidas son frutos de una evaluación hecha a los estudiantes del $3^{\circ}$ curso de la Educación Media. "Las evaluaciones nacionales tienen como propósito principal monitorear el logro de los objetivos curriculares, mejorar el logro académico de los estudiantes y fortalecer el sistema educativo" (Modulo I, p. 5).

Por lo tanto, tener una definición de evaluación de los aprendizajes en la actualidad, es de suma importancia, pues existe tantas, que se vuelven complejas enunciarlas. Pero, aquí se 
selecciona conceptos que presentan un progreso paulatino a través del tiempo, hasta llegar a la taxonomía de Bloom.

\section{ESTRATEGIAS METODOLÓGICAS O MATERIALES Y MÉTODOS}

La investigación busca establecer las variables objeto de estudio como sugiere Hernández Sampieri (2014) que los estudios descriptivos son útiles para mostrar con precisión los ángulos o dimensiones de un fenómeno, suceso, comunidad, contexto o situación.

En esta clase de estudios el investigador debe ser capaz de definir, o al menos visualizar, qué se medirá (qué conceptos, variables, componentes, etc.) y sobre qué o quiénes se recolectarán los datos (personas, grupos, comunidades, objetos, animales, hechos). Por ejemplo, si vamos a medir variables en escuelas, es necesario indicar qué tipos habremos de incluir (públicas, privadas, administradas por religiosos, laicas, de cierta orientación pedagógica, de un género u otro, mixtas, etc.). Si vamos a recolectar datos sobre materiales pétreos, debemos señalar cuáles. La descripción puede ser más o menos profunda, aunque en cualquier caso se basa en la medición de uno o más atributos del fenómeno de interés (p.92).

El tipo de estudio utilizado será la del paradigma Cuali-Cuantitativo que según (Martens, 2005), es la combinación de los enfoques cuantitativo y cualitativo y pueden involucrar la conversión de datos cuantitativos en cualitativos y viceversa.

Para localizar los documentos bibliográficos necesarios se utilizaron varias fuentes de búsqueda. Como academia.edu, utilizando descriptores: evaluación, evaluación de los aprendizajes, taxonomía de Bloom, revista de artículos científicos. La referencia principal de los resultados de SNEPE 2015 fueron, el Informe Nacional por Eje Temático evaluación censal 2015 que el MEC dio a cada institución donde se aplicaron las pruebas, los resultados que están al acceso del público en la página web del Ministerio de Educación y Ciencias; el libro de Lengua Castellana y Literatura del $3^{\circ}$ curso de la Media, contrastando los trabajos que realizan los estudiantes en clase con las pruebas presentadas. Igualmente, se utilizó internet buscando por "Google académico" utilizando los mismos descriptores.

Se seleccionaron documentos que contenían información sobre taxonomía de Bloom, lectura crítica sobre temas de evaluación, estadísticas de resultados de las evaluaciones y resúmenes.

\section{Esclarecimiento de la Noción de Evaluación de los Aprendizajes}


Castillo Arredondo, y Cabrerizo Diago (2010) citando a Tyler (1950), dice "El proceso de evaluación es esencialmente el proceso de determinar hasta qué punto los objetivos educativos han sido actualmente alcanzados mediante los programas y currículos de enseñanza" (p. 5).

Para Forns (1980), el concepto de evaluación se puede abordar en varios niveles:

1. A nivel técnico, en cuanto que la evaluación se dirige a comprobar el funcionamiento del sistema educativo: se trata de un control o balance que indica si el sistema educativo está cumpliendo sus funciones.

2. A nivel ideológico, en el que la evaluación tiene dos funciones importantes y delicadas: Por una parte, "legitimar" la herencia cultural, ayudando de este modo a perpetuar el orden establecido; y por otra, "eliminar" a los sujetos que no pertenecen a la clase social dominante, ya que no han asimilado debidamente los principios ideológicos que se le pretendían transmitir.

3. A nivel psicopedagógico, ya que se aplica a alumnos concretos más que a entidades.

Mientras que, Ander Egg (2000) asevera que, “La evaluación es una forma de investigación social aplicada, sistemática, planificada y dirigida; encaminada a identificar, obtener y proporcionar de manera válida y fiable, datos e información suficiente y relevante en que apoyar un juicio acerca del mérito y el valor de los diferentes componentes de un programa o de un conjunto de actividades específicas que se realizan, han realizado o realizarán, con el propósito de producir efectos y resultados concretos" (p. 47).

Por otro, Castillo Arredondo (2002) subraya que "La evaluación debe permitir, por un lado, adaptar la actuación educativo-docente a las características individuales de los alumnos a lo largo de su proceso de aprendizaje; y por otro, comprobar y determinar si estos han conseguido las finalidades y metas educativas que son el objeto y la razón de ser de la actuación educativa" (p. 7).

No obstante, Valverde (2017), aludiendo a Mora (2004) quien afirma que, la evaluación es de utilidad si va encaminada hacia el progreso donde se identifican los puntos débiles y fuertes con la idea de mejorar. Así mismo, ella expresa que es necesario tomar en cuenta la viabilidad y la equidad (p.76).

En cambio, Serpa Naya (2008) enfatiza que "La evaluación es un seguimiento continuo y sistemático que se le hace para identificar los logros y las dificultades presentadas en el proceso y poder tomar decisiones que lleven a un mejoramiento de la calidad educativa". 
Sin embargo, Castillo Arredondo, S. et al. (2010) reiterando a Hancones (2005), habla de un "Proceso por medio del cual los profesores buscan y usan información procedente de diversas fuentes para llegar a un juicio de valor sobre el alumno o sistema de enseñanza en general o sobre alguna faceta particular del mismo" (p.7).

A propósito, para D’agostino (2007) "la evaluación educativa es aquel proceso orientado hacia la determinación, búsqueda y obtención de evidencias acerca del grado y nivel de calidad del aprendizaje del estudiante, para juzgar si es adecuado o no y tomar las medidas correspondientes" (p.12).

Asimismo, Castillo Arredondo, S. et al. (2010) destaca lo siguiente:

La evaluación educativa está adquiriendo cada vez mayor relevancia y determinación en la enseñanza. Hasta ahora, esta ha sido una actividad al término de un periodo de enseñanza en la explicación de una materia para comprobar el nivel de aprendizaje adquirido por los alumnos durante dicho periodo. En la actualidad, la evaluación educativa está llamada a ser el eje integrador, vertebrador y dinamizador de los procesos de enseñanza y de aprendizaje: los datos aportados por la evaluación educativa le pueden permitir al estudiante tener una nueva oportunidad para aprender lo que hasta entonces no había logrado; y al profesor una nueva ocasión para volver a enseñar aquello que los estudiantes no alcanzaron a comprender con las explicaciones anteriores (p.16).

En suma, se podría decir que la evaluación de los aprendizajes es un proceso por el cual se puede recolectar información para verificar si se ha logrado los objetivos esperados de un programa o currículo de enseñanza y obtener evidencias del grado de nivel de calidad del aprendizaje del estudiante para tomar decisiones que lleven al mejoramiento de la calidad educativa.

Que es la taxonomía de Bloom

La Revista Eduteka en su artículo sobre las preguntas de elección múltiple y la Taxonomía de Bloom, narra brevemente quién fue Benjamín Bloom:

Al terminar la Convención de la Asociación Norteamericana de Sicología en 1.948, Benjamín Bloom lideró la formulación de una clasificación de "Los Objetivos del Proceso Educativo". Se identificaron Tres "dominios" de actividades de aprendizaje. El primero de ellos, denominado Dominio Cognitivo, supone el conocimiento y desarrollo de habilidades y actitudes intelectuales. (Los otros dos dominios son el Afectivo y el Psicomotor de los que no vamos a ocuparnos en esta oportunidad) Eventualmente, Bloom y sus colaboradores 
establecieron una jerarquía de Objetivos de Aprendizaje, a la que comúnmente se refiere la gente como Taxonomía de Bloom, que propone dividir los objetivos cognitivos en subcategorías organizadas del comportamiento más simple al más complejo (p.1).

Veamos cuales son las propuestas que nos propone Bloom en su taxonomía:

Conocimiento: El sujeto es capaz de recordar información anteriormente aprendida. Reconoce información, ideas, hechos, fechas, nombres, símbolos, definiciones, etc., de una forma aproximada a cómo los ha aprendido, algunos ejemplos: escribir, reproducir, etiquetar, definir, recordar.

Comprensión: El sujeto entiende "Se hace suyo" aquello que ha aprendido y esto lo demuestra cuando es capaz de presentar la información de otra manera, cuando la transforma, cuando encuentre relaciones con otra información de otra manera, cuando se asocia a otro hecho, cuando se saben decir las posibles causas y consecuencias, ejemplos: ordenar, seleccionar, dar ejemplos, explicar, parafrasear, exponer.

Aplicación: El sujeto es capaz de utilizar aquello que ha aprendido. Cuando aplica las destrezas adquiridas a nuevas situaciones que se le presenten. Cuando utiliza la información recibida en situaciones nuevas y concretas para resolver problemas, por ejemplo: construir, relacionar, solucionar, transferir, demostrar.

Análisis: Cuando el sujeto es capaz de descomponer el todo en sus partes y puede solucionar problemas a partir del conocimiento adquirido. Cuando intenta entender la estructura de la organización del material informativo examinando las partes de las cuáles se compone. Analizar, discriminar, contrastar, diagramar, valorar, cuestionar, experimentar.

Síntesis: Cuando el sujeto es capaz de crear, integrar, combinar ideas, planear, proponer nuevas maneras de hacer. Crear aplicando el conocimiento y habilidades anteriores para producir alguna cosa nueva y original (adaptar, categorizar, recopilar, inventar, formular). Es el proceso de trabajar con fragmentos, partes, elementos, organizarlos, ordenarlos y combinarlos para formar un todo, un esquema o estructura que antes no estaba presente de manera clara. Los resultados del aprendizaje en esta área enfatizan comportamientos creativos dando mayor importancia a la formulación de nuevos patrones o estructuras (p. 2-5).

En la actualidad, Aliaga Olivera (2012), subraya que la taxonomía de Bloom pasó por dos actualizaciones: 
En el 2000 sufrió una revisión por uno de sus discípulos quien, para cada categoría, cambió tanto el uso de sustantivos por verbos, como su secuencia. Recientemente, el doctor Andrew Churches actualizó dicha revisión para ponerla a tono con las nuevas realidades de la era digital. En ella, complementó cada categoría con verbos y herramientas del mundo digital que posibilitan el desarrollo de habilidades para Recordar, Comprender, Aplicar, Analizar, Evaluar y Crear (p. 3).

Pero esto no es todo, existen detractores que no están de acuerdo totalmente con los postulados por Bloom en su taxonomía porque dicen que ya está desfasada en la actualidad. Por ejemplo, Rodríguez Ariza (2018), en su artículo Crítica a la taxonomía de Bloom, cuestiona dicha taxonomía diciendo que:

Los avances tecnológicos en el estudio del cerebro, y el surgimiento de la neurociencia cognitiva y otras evidencias hacen poco viable mantener su sustento epistemológico. Las críticas son:

1. Se basan en los principios conductistas que predominaba a principios de siglos en la psicología. Un dato adicional, es que la taxonomía surge en los comienzos de la revolución de las ciencias cognitivas a mediados del siglo XX. Por tanto, son 60 años en el cual se ha avanzado mucho en la comprensión de la naturaleza del aprendizaje humano en diversos campos.

2. Tiende a una fragmentación del currículo y eso lleva a una falta de visión integral de los objetivos del sistema educativo. Se da entender que los docentes deben enfatizar primero en la memorización de todo el currículum antes que los estudiantes puedan comprender o aplicar el conocimiento. Ya que se concibe el proceso educativo como predeterminado y mecanicista (Aubrey y Riley, 2016), fomentadora de la homogeneización y la pasividad (Gimeno Sacristán).

3. Se ha caído en el error de considerar la taxonomía una teoría sobre el aprendizaje. Como si la taxonomía pretendiera explicar cómo aprenden los seres humanos. La taxonomía sirve para planificar y evaluar, no para explicar. En estas últimas dos décadas la neurociencia cognitiva ha aportado valiosos conocimientos sobre la lectura, la escritura, la dislexia, la discalculia, las emociones, los tipos de memoria, entre otros.

4. La ambigüedad y la falta de mayor precisión en algunos conceptos como “conocimiento" o "recordar". Por ejemplo, en el caso de recordar, es decir, la memoria, se describe en la taxonomía como si esta fuera una sola. En cambio, la neurociencia cognitiva 
ha identificado diversos tipos de memorias que funcionan de forma independiente e interrelacionadas: (a) la memoria inmediata, (b) la memoria operativa y (c) las memorias a largo plazo (episódica, semántica, procedimental entre otras) (Carrillo-Mora,2010). Todas ellas muy activas en el proceso de aprendizaje de una persona.

Ahora bien, veamos cuales son los contenidos curriculares que son evaluados en la prueba SNEPE en Lengua Castellana y Literatura del tercer curso de la Educación Media.

La siguiente tabla muestra la distribución de los contenidos del área estudiados a lo largo la Educación Media que son evaluados en Lengua castellana y Literatura presentada en el Informe Nacional Analítico Evaluación Censal 2015:

Evolución de la literatura, corrientes y movimientos literarios del Siglo XX (Contexto histórico, social y filosófico de la obra. Rasgos psicológicos y culturales presentes en la obra: creencias, costumbres, emociones, modos pensar, valores, esperanzas, conflictos de carácter religioso, moral y político).

Vocabulario y figuras literarias o recursos léxico: (Significado de palabras y expresiones. Sinonimia, antonimia, homonimia, paronimia y campo semántico. Sinestesia, metáfora, antítesis, hipérbole, metonimia, ironía repetición, personificación).

Contenido y relación en texto: Superestructura (Tipología textual. Organización discursiva del texto. Intención comunicativa y situación comunicacional. Funciones y niveles del lenguaje).

Macroestructura (Características de personajes, sus acciones, tiempo y lugar de la acción. Secuencia de acciones o ideas en relación con las ideas expresadas por el autor. Ideas principal y secundaria de párrafos, idea central de texto, deducción de ideas, tema, mensajes. Causa, efecto y consecuencia en relación con las ideas expresadas por el autor). Relaciones morfosintácticas y ortográficas: Concordancia nominal y verbal. Vicios del lenguaje: cacofonía, dequeísmo. Procedimiento de cohesión textual: uso de conectores. Relaciones de referencia endofórica (anáfora y catáfora), exofórica (deixis). Normativa de la lengua: literal, acentual y puntual (p. 28).

En cambio, los contenidos desarrollados en el libro de Lengua Castellana y Literatura del $3^{\circ}$ de la Media, son:

El hombre y su sed de conocimiento. El tema del "libre Albedrío". La guerra y la paz en la literatura. Conflicto a partir del contacto de culturas. Comienzo y fin del ser humano; esta unidad trata sobre el modernismo y tecnología. 
De las capacidades a desarrollar en todo el libro, cinco unidades o ejes temáticos, tienen dos capacidades iguales: 1- Analiza los elementos estéticos utilizados en obras literarias paraguayas y universales. 2- Identifica los rasgos socioculturales presentes en las obras literarias paraguayas y universales. Las demás capacidades varían de acuerdo al tema a desarrollar en cada unidad.

\section{RESULTADOS Y DISCUSIÓN}

Los resultados obtenidos de las pruebas SNEPE 2015 en Lengua Castellana permiten ver cuáles fueron los logros de los estudiantes en dicha evaluación.

De 100 estudiantes examinados, cero (0) estudiantes llegaron al nivel IV; cinco (5) hasta el nivel III; once (11) estudiantes lograron hasta el nivel II; y ochenta y cuatro (84) realizaron sin dificultad los ejercicios del nivel I.

Las derivaciones resultantes por eje temático demuestran el nivel de comprensión de los estudiantes en un proceso de lectura para realizar las tareas correspondientes. En el cuadro se visualiza el resultado de las evaluaciones por eje temático y la calificación obtenida en cada una de ello.

\begin{tabular}{|l|l|l|l|l|}
\hline \multicolumn{1}{|c|}{ CALIFICACIÓN } & 1 & 2 & 3 & 4 y 5 \\
\hline $\begin{array}{l}\text { Comprensión de la expresión estética, estilos } \\
\text { lingüísticos y aprehensión de realidades culturales } \\
\text { (evolución de la literatura, clásica, edad media, } \\
\text { renacimiento y otros). }\end{array}$ & 63 & 21 & 0 & 16 \\
\hline $\begin{array}{l}\text { Vocabulario y figuras literarias (significados de } \\
\text { palabras y expresiones). }\end{array}$ & 95 & 5 & 0 & 0 \\
\hline $\begin{array}{l}\text { Superestructura (tipología textual, textos científicos, } \\
\text { periodísticos, informativos, genero literarios, otros). }\end{array}$ & 100 & 0 & 0 & 0 \\
\hline $\begin{array}{l}\text { Macroestructura (características de personajes, y sus } \\
\text { acciones). }\end{array}$ & 84 & 11 & 5 & 0 \\
\hline $\begin{array}{l}\text { Comprensión lingüística (usos correctos de adverbios, } \\
\text { pronombres, preposiciones, escritura correcta, otros). }\end{array}$ & 100 & 0 & 0 & 0 \\
\hline
\end{tabular}

Fuente N. ${ }^{\circ}$ 1: Puntaje obtenido por eje temático según resultado. Elaboración propia.

Estos datos indican que el nivel de desempeño académico de los estudiantes del tercer curso de la Media está por debajo de los estándares propuestos por el MEC en dicha evaluación. 
Con estos resultados, se puede inferir varios postulados. Por un lado, que la educación que reciben los estudiantes no es de calidad. Que las estrategias utilizadas por los docentes para enseñar a sus alumnos están desfasadas. Hace falta que los docentes tengan una formación permanente. Que los materiales didácticos utilizados por los estudiantes no están de acorde a la finalidad educativa que se pretende en la actualidad en nuestro país. En cambio, el postulado que más se pondera en los medios y algún representante del MEC, que la culpa del fracaso escolar, los responsables directos son los docentes, pero sin ningún fundamento valido.

Otro punto importante, que no se puede dejar de lado es, cuál es la finalidad de estas pruebas, para qué se aplican. Qué pretende el MEC con evaluar a los estudiantes, o es solamente para conocer el nivel de logro académico.

Según el MEC, tiene como objetivo general "disponer información del nivel de desempeño de los estudiantes y de factores asociados al aprendizaje que retroalimenten la política educativa".

A esto, le respondemos con palabras de Castillo Arredondo que parafraseando a Tyler dice que "la evaluación es esencialmente el proceso de determinar hasta qué punto los objetivos educativos han sido actualmente alcanzados mediante los programas y currículos de enseñanza".

Sigue diciendo Castillo Arredondo que, "la evaluación debe permitir, por un lado, adaptar la actuación educativo-docente a las características individuales de los alumnos a lo largo de su proceso de aprendizaje".

Y que en "la actualidad, la evaluación educativa está llamada a ser el eje integrador, vertebrador y dinamizador de los procesos de enseñanza y de aprendizaje".

Pero, por otra parte, el MEC aclara que "Las evaluaciones nacionales tienen como propósito principal monitorear el logro de los objetivos curriculares, mejorar el logro académico de los estudiantes y fortalecer el sistema educativo".

Si aplicamos lo dicho por Forns (1980) "que la evaluación se dirige a comprobar el funcionamiento del sistema educativo". Iría en relación con lo planteado por el MEC, de fortalecer el sistema educativo.

En esto hace hincapié Ander Egg (2000) que, "la evaluación es una forma de investigación social aplicada, sistemática, planificada y dirigida; encaminada a identificar, obtener y 
proporcionar de manera válida y fiable datos e información suficiente con el propósito de producir efectos y resultados concretos".

De esto se puede inferir que, las evaluaciones hechas necesariamente tienen un propósito para lo cual se aplica. Y queda claro que es para monitorear el logro de los objetivos curriculares, mejorar el logro académico de los estudiantes y fortalecer el sistema educativo. Con los resultados obtenidos se cuenta con los elementos necesarios para tomar las posturas necesarias y reorganizar el sistema educativo para que se una educación de calidad en nuestro país. Luego, verificar de los avances o retrocesos en la próxima evaluación. No sirve de nada esperar otras pruebas para tomar acciones.

Pero, cual fue el motivo para optar por este estilo de pruebas considerada SNEPE, que se basan en la Taxonomía de Bloom.

Como se vio, la taxonomía de Bloom organiza "los objetivos del proceso educativo" en niveles y tienen una estructura jerárquica que va desde lo más simple a lo más complejo. Es decir, las pruebas comienzan con lo más fácil, luego va aumentando la complejidad hasta llegar al nivel IV que es lo más complicado. Pero, para esto, los alumnos tienen que tener conocimiento de cómo serán las pruebas.

El libro de texto de Lengua Castellana y Literatura debe ser un ejemplo de estas prescripciones, donde los estudiantes entrarán en contacto directo con los objetivos curriculares y las capacidades básicas que los alumnos tienen que asimilar al final del ciclo de enseñanza.

Ahora bien, al analizar el libro de texto, se verificó que los ejercicios de fijación del conocimiento, presentan niveles de complejidad I y II en todos los ejes temáticos. Pero, se centran en explicaciones y comentarios.

En cambio, los ejercicios de las pruebas SNEPE son presentados en forma de selección múltiple con distractores correspondientes en donde el estudiante debe de identificar cual es la respuesta válida.

Esto hace que el resultado de las pruebas SNEPE de un resultado negativo, a simple vista. Pero, como se nota, existen factores para que se den estas consecuencias.

En este sentido, las pruebas tomadas serían de gran ayuda para mejorar el sistema educativo paraguayo. Acentuó lo dicho, parafraseando a Serpa Naya (2008) subrayando que "la evaluación es un seguimiento continuo y sistemático que se hace para identificar los logros, 
las dificultades presentadas y tomar decisiones que lleven a un mejoramiento de la calidad educativa".

Los resultados de las pruebas estandarizadas tienen que facilitar los medios para que la educación mejore. Como dice Castillo Arredondo, y Cabrerizo Diago (2010) "en la actualidad, la evaluación educativa está llamada a ser el eje integrador, vertebrador y dinamizador de los procesos de enseñanza y de aprendizaje". Esta afirmación nos ilumina el horizonte a seguir, a la vez, de cómo utilizar los datos proporcionados por las evaluaciones estandarizadas aplicadas para medir el conocimiento de los alumnos.

Es necesario en base a las evidencias obtenidas que el libro de texto cambie de estructura y todos los ejes temáticos sean elaborados y presentados según la taxonomía de Bloom, exhibiendo los objetivos cognitivos en subcategorías organizadas del más simple al más complejo.

A pesar de que existen críticas a la taxonomía de Bloom por las "ambigüedades de algunos conceptos". Las taxonomías ayudan a organizar el aprendizaje de los estudiantes y la asimilación del conocimiento. En consecuencia, su aplicación en el proceso de enseñanzaaprendizaje-evaluación, para mejorar y elevar la calidad educativa en el país es factible.

\section{CONCLUSIÓN O CONSIDERACIONES FINALES}

Con el análisis hecho, se desglosa meramente que no existe una relación de concordancia y continuidad entre lo desarrollado en el libro de texto de Lengua Castellana y Literatura y las pruebas SNEPE.

Por otro, los ejercicios que contiene el libro de texto, no están hechas según la taxonomía de Bloom. Tampoco, como presentan las evaluaciones de SNEPE.

Debido a esto, tenemos malos resultados en las evaluaciones hechas y se evidencia la mala calidad de nuestra educación escolar. En este punto, no vale la pena buscar culpables, ni aludir a supuestos que los docentes son los responsables de que se tenga resultados negativos por falta de una actualización didáctica.

En este punto, es oportuno recordar que uno los objetivos de las evaluaciones nacionales es mejorar el logro académico de los estudiantes y fortalecer el sistema educativo. Por tanto, es menester del Ministerio de Educación y Ciencias utilizar los datos obtenidos en las pruebas para actualizar y adecuar los materiales didácticos acorde a los delineamentos utilizados para implementar las pruebas SNEPE. Utilizando la taxonomía de Bloom en la hora de programar los planes y programas de educación. 
Con esto, se corta la brecha que se demostró. Que los trabajos de fijación de conocimiento de los textos de lectura no condicen con las evaluaciones tomadas.

El MEC como órgano rector de la educación en el Paraguay tiene la potestad de realizar los cambios pertinentes para que se den las circunstancias y haya una educación de calidad en nuestro país. No basta con enviar materiales de informes en las instituciones donde se llevaron a cabo las evaluaciones y sugerir a los directores los lineamientos para la elaboración de planes de mejora institucional de acuerdo a los resultados obtenidos, que es una quimera. Para que los docentes implementen en los planeamientos educativos los planes de mejora institucional, todo el corpus tendría que salir del MEC para que sea acatado por todas las instituciones escolares.

Porque, artículo $3^{\circ}$ de la ley 1264/98 subraya que: "El Estado garantizará el derecho de aprender y la igualdad de oportunidades de acceder a los conocimientos y a los beneficios de la cultura humanística, de la ciencia y de la tecnología, sin discriminación alguna. Garantizará igualmente la libertad de enseñar, sin más requisitos que la idoneidad y la integridad ética, el derecho a la educación religiosa y al pluralismo ideológico". Por esta cuestión, prima que el Ministerio de Educación y Ciencias tome la iniciativa que los planes de mejora institucional lleguen a todas las instituciones educativas y no solamente donde se llevaron a cabo las pruebas. Caso contrario, no se cumple lo estipulado por la ley.

Aún más, esto implica que la educación se debe replantear por completo. Comenzando desde la formación inicial de los futuros docentes, que tienen que recibir formación con este estilo realizando sus prácticas y planeamientos educativos con el modelo que presenta la taxonomía de Bloom, para que luego lleven a la práctica en el momento de ejercer la docencia.

Además, para que todo el plantel docente sea formado y actualizado, es necesario utilizar la formación continua de profesores, para que todos lleguen a conocer y aplicar las nuevas estrategias de enseñanza postuladas la política educativa del gobierno por medio del MEC. Porque muchos docentes se formaron a finales del siglo pasado y no tienen conocimiento de estas estrategias, ya que, no formaban parte de la política educativa del país.

\section{LISTA DE REFERENCIAS}

Aliaga Olivera, S. (2012). Universidad Cesar Vallejo.

https://santiagowalteraliagaolivera.files.wordpress.com/2012/03/4-taxonomiade-bloom1.pdf 
Ander- Egg, E. (2000): Metodología y práctica de la Animación Sociocultural. CCS. Madrid.

Castillo Arredondo, S. (2002) (coord.): Compromisos de la evaluación educativa. Madrid. Pearson Educación.

Castillo Arredondo, S. y Cabrerizo Diago, J. (2010). Evaluación educativa de aprendizajes y competencias. PEARSON EDUCACIÓN, S.A. Madrid.

D’agostino, Giuseppa. (2007). Aspectos teóricos de la evaluación educacional.

Recuperado de:

https://books.google.com.ni/books?id=8ueTibkvwtwC\&pg=PA17\&dq=definici $\% \mathrm{C} 3 \% \mathrm{~B} 3 \mathrm{n}+\mathrm{de}+$ evaluaci\%C3\%B3n+educativa\&hl=es419\&sa=X\&ved=0ahUKE wjAjamY1cTSAhUqw1QKHaNaCp8Q6AEIHjAB\#v=onepage \&q\&f=false

EDUTEKA. Las preguntas de elección múltiple y la Taxonomía de Bloom

Forns, M. (1980): La Evaluación de los aprendizajes. En Coll, C.: Áreas de intervención de la Psicología. Barcelona. Horsori.

Hernández Sampieri, R. (2014). Metodología de la Investigación Científica $6^{\text {a }}$ ed. México D.F. Mc Graw Hill Education.

http://www.eduteka.org/articulos/EleccionMultiple

Ley General de Educación - Paraguay (1998) PODER LEGISLATIVO LEY No 1264 GENERAL DE EDUCACIÓN.

MEC (2016). INFORME NACIONAL ANALÍTICO. Resultados de la primera evaluación censal de logros académicos a estudiantes de finales de ciclo y nivel. Evaluación censal 2015. Resultados Cognitivos.

MEC (2016). Lengua Castellana y Literatura. Texto para el estudiante $3^{\circ}$ curso de la Media plan común.

MEC (2016). Modulo I: Uso de resultados de las pruebas de aprendizajes del SNEPE en instituciones educativas.

MEC (2018). ¿Cuál es logro académico de los estudiantes? Resultados educativos informe institucional. Evaluación Censal 2015.

MEC (2019). Programa de evaluación censal de logros académicos a estudiantes de finales de ciclo/nivel y de factores asociables al aprendizaje. Instituto Nacional de Evaluación Educativa. Dirección de Evaluación de Logros de Aprendizajes Curriculares. 
Rodríguez Ariza, C. (2018). Aprendiendo a Aprender para el Desarrollo.

https://triplead.blog/2018/02/16/criticas-a-la-taxonomia-de-bloom/

Serpa Naya (2008): http://cursosunad.blogspot.com/2008/06/definicin-de-

evaluacin.html

Valverde, X. (2017). La evaluación tradicional vs. evaluación alternativa en la FAREMCarazo. Revista Torreón Universitario, 15 (6), 75-82. Recuperado de:

www.faremcarazo.unan.edu.ni 